\title{
Deconvoluting Lipid Nanoparticle Structure for Messenger RNA Delivery
}

\author{
Yulia Eygeris, ${ }^{\dagger}$ Siddharth Patel, ${ }^{\dagger}$ Antony Jozic, ${ }^{\dagger}$ and Gaurav Sahay ${ }^{*, \dagger, \ddagger}$ \\ $\dagger$ Department of Pharmaceutical Sciences, College of Pharmacy, Oregon State University, \\ Portland, Oregon 97201 \\ $\ddagger$ Department of Biomedical Engineering, Oregon Health $\&$ Science University, Portland, \\ Oregon 97201 \\ E-mail: sahay@ohsu.edu \\ Phone: (503) 346-4698
}




\section{Supporting Information Available}

\section{Materials and Methods}

Materials. CleanCap Firefly luciferase (FLuc) mRNA was purchased from TriLink Biotechnologies (San Diego, CA). [(6Z,9Z,28Z,31Z)-heptatriaconta-6,9,28,31-tetraen-19-yl] 4-(dimethylamino)butanoate (DLin-MC3-DMA) was custom synthesized by Biofine (Blaine, WA); 1,2-distearoyl-sn-glycero-3-phosphocholine (18:0 PC, DSPC) was purchased from Avanti Polar Lipids, Inc, (Alabaster, AL); 1,2-dimyristoyl-sn-glycero-3-methoxypolyethylene glycol 2000 (Sunbright DMG-PEG2000) was purchased from NOF Corporation America (White Plains, NY); cholesterol, $\beta$-sitosterol, fucosterol, 1-(4-trimethylammonium-phenyl)6-phenyl-1,3,5-hexatriene (TMA-DPH) were purchased from Sigma Aldrich (St Louis, MO); stigmastanol and campesterol were purchased from Cayman Chemicals (Ann Arbor, Michigan); Quant-iT RiboGreen ${ }^{\mathrm{TM}}$ RNA reagent, CellTiter-Fluor ${ }^{\mathrm{TM}}$ Cell Viability and ONE$\mathrm{Glo}^{\mathrm{TM}}$ Luciferase assays, HyClone deionized water, PBS buffer, and ethanol were purchased from Fisher Scientific (Federal Way, WA). Lipid nanoparticles were prepared using Nanoassemblr Benchtop microfluidic mixer (Precision Nanosystems, Vancouver, BC). Nanoparticle size and polydispersity were characterized using Malvern Zetasizer ZS (Malvern Panalytical Inc., Westborough MA). Fluorescence was recorded using Jasco FP-8200 spectrofluorometer (Easton, MD). Thermal analysis was performed using a differential scanning calorimeter (TA Instruments, New Castle, DE). Quantifoil R1.2/1.3 300-mesh grids (Ted Pella, Redding, CA), FEI Vitrobot and FEI Titan Krios (ThermoFisher, Hillsboro, OR), copper washers, leaf springs, and grid boxes (Subangstrom, Brooklyn, NY) were used for cryoEM sample prep, storage, and imaging. All cell culture reagents were purchased from Corning. Prism (GraphPad) was used for data visualization.

Nanoparticle preparation and characterization. Lipid nanoparticles were prepared by mi-

crofluidic mixing using the previously described method. ${ }^{1,2}$ Briefly, a lipid solution in ethanol (containing DLin-MC3-DMA, DSPC, a sterol, and DMG-PEG2000 at the molar ratios of 
50:10:38.5:1.5, respectively, and total concentration of $5.5 \mathrm{mM}$ and 20-fold excess by mass compared to mRNA) was combined with an aqueous mix containing mRNA, water, and $100 \mathrm{mM}$ pH 4 citrate buffer (3-fold excess by volume compared to lipid solution) using a microfluidic mixer. For DSC, the formulation did not contain any mRNA and its volume was replaced with equivalent volume of DI water. Nanoparticles were then dialyzed twice against $3 \mathrm{~L}$ of pH 7 PBS buffer and concentrated using appropriate centrifuge filters. Nanoparticle size was determined using sufficiently diluted solutions in PBS via dynamic light scattering (DLS), and mRNA encapsulation efficiency was determined using a modified Quant-iT RiboGreen RNA reagent (Invitrogen).

In Vitro transfection. Cells were plated in white, clear-bottom 96-well plates. HeLa cells were plated at 4,000 cells/well and transfected 24 hours after initial plating with a known quantity of mRNA. The transfection results were then read 24h later using CellTiter-Fluor Cell Viability and ONE-Glo Luciferase Assays (Promega).

Cryo-electron microscopy. CryoEM acquisition was performed at $300 \mathrm{kV}$ using Falcon III and K3 cameras with DED. 3-5 ul of the sample was dispensed on a plasma cleaned grid in the Vitrobot chamber at $95 \%$ relative humidity and allowed to rest for 30 seconds. Then, the grid was blotted for 3 seconds with filter paper and plunged into liquid ethane cooled by liquid nitrogen. The frozen grids were then checked for visible defects and assembled into cassettes. The collected images were then processed and analyzed manually using Fiji. ${ }^{3}$ LNP populations contained at least 85 particles. Only unobstructed LNPs with clearly defined edges were included in the statistical analysis to ensure accurate classification of LNP populations and to avoid counting structural deformities due to sample prep or ice thickness issues. Polydispersity index (PDI) was calculated as $P d I=(\sigma / \mu)^{2}$, where $\sigma$ standard deviation, $\mu$ - mean of particle size.

Thermal analysis. Differential scanning calorimetry (DSC) was performed using formulations containing no mRNA and either DSC Q2000 or 2920 instrument (TA Instruments) in $0-100^{\circ} \mathrm{C}$ range at $10{ }^{\circ} \mathrm{C} / \mathrm{min}$. The collected data was processed by subtracting PBS 
background signal and analyzed using TA Universal Analysis software.

Membrane fluidity assay. TMA-DPH assay was performed as described elsewhere. ${ }^{4}$ Briefly, LNP solution was diluted to a total lipid concentration of $0.5 \mathrm{mg} / \mathrm{ml}$ and mixed in a microcuvette with equal volume of TMA-DPH, diluted from $1 \mathrm{mM}$ stock solution in EtOH to $0.25-2 \mathrm{ug} / \mathrm{ml}$ final concentration (ca. 100-700 lipid:fluorophore ratio) with $\mathrm{pH} 7$ PBS buffer. The fluorescence anisotropy was recorded using temperature-controlled automatic polarization setup at wavelengths $355 \mathrm{~nm}$ (excitation) and $430 \mathrm{~nm}$ (emission), ramping the temperature at $5^{\circ} \mathrm{C} / \mathrm{min}$.

\section{Author contributions}

Prof. G. Sahay conceived the idea, directed the research, and reviewed the paper. Dr. Y. Eygeris wrote the paper, formulated the particles, performed transfection study, conducted the cryoEM imaging and the TMA-DPH assay, and analyzed the DSC data. S. Patel conceived the idea of TMA-DPH experiments, formulated the particles, and revised the paper. A. Jozic conducted DSC experiments. 


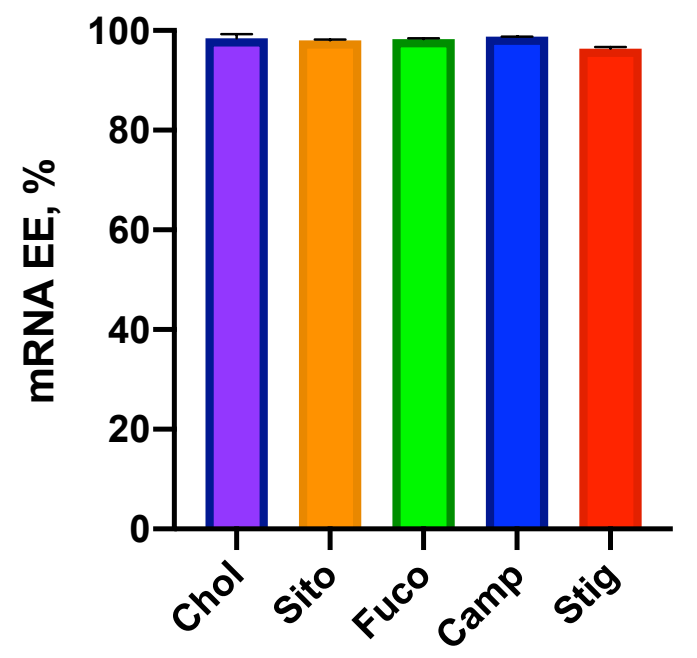

Figure S1: mRNA encapsulation efficiency for the phytosterol formulations $(n=6)$.

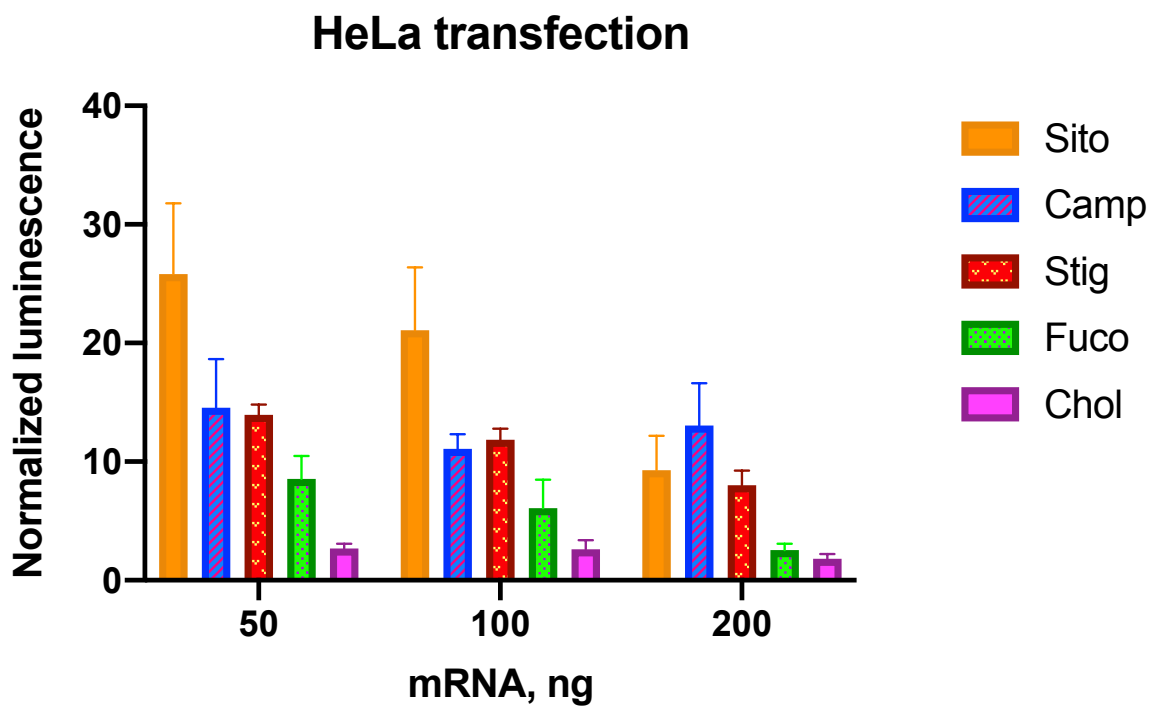

Figure S2: HeLa transfection efficiency data $(n=3)$. Luminescence values were normalized by the cell counts. 

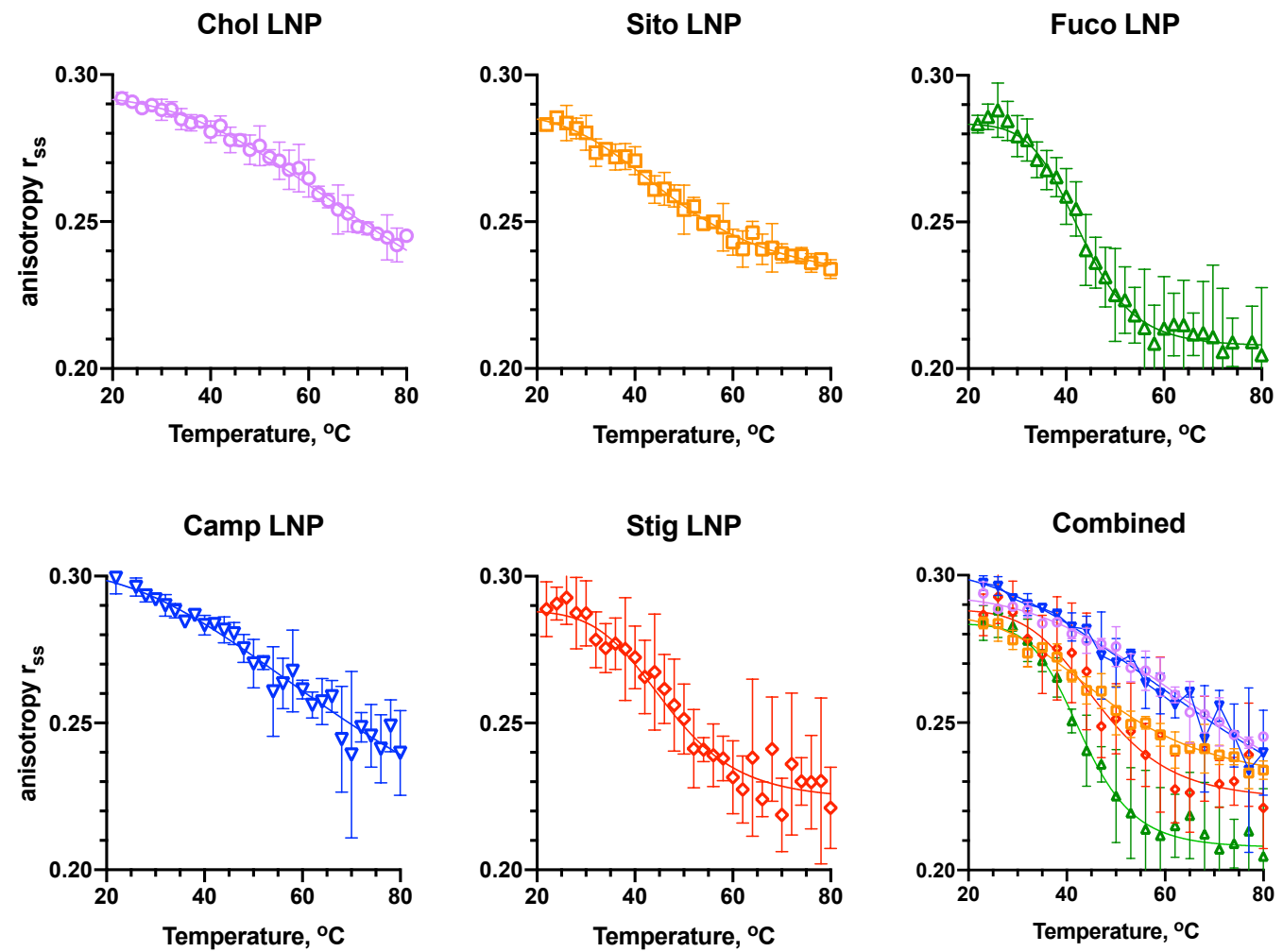

Figure S3: TMA-DPH assay results. Decrease of the mean and increase of the standard deviation of the anisotropies above $40^{\circ} \mathrm{C}$ is likely caused by the increased Brownian motion of the probe and the lipids $(n=4)$.

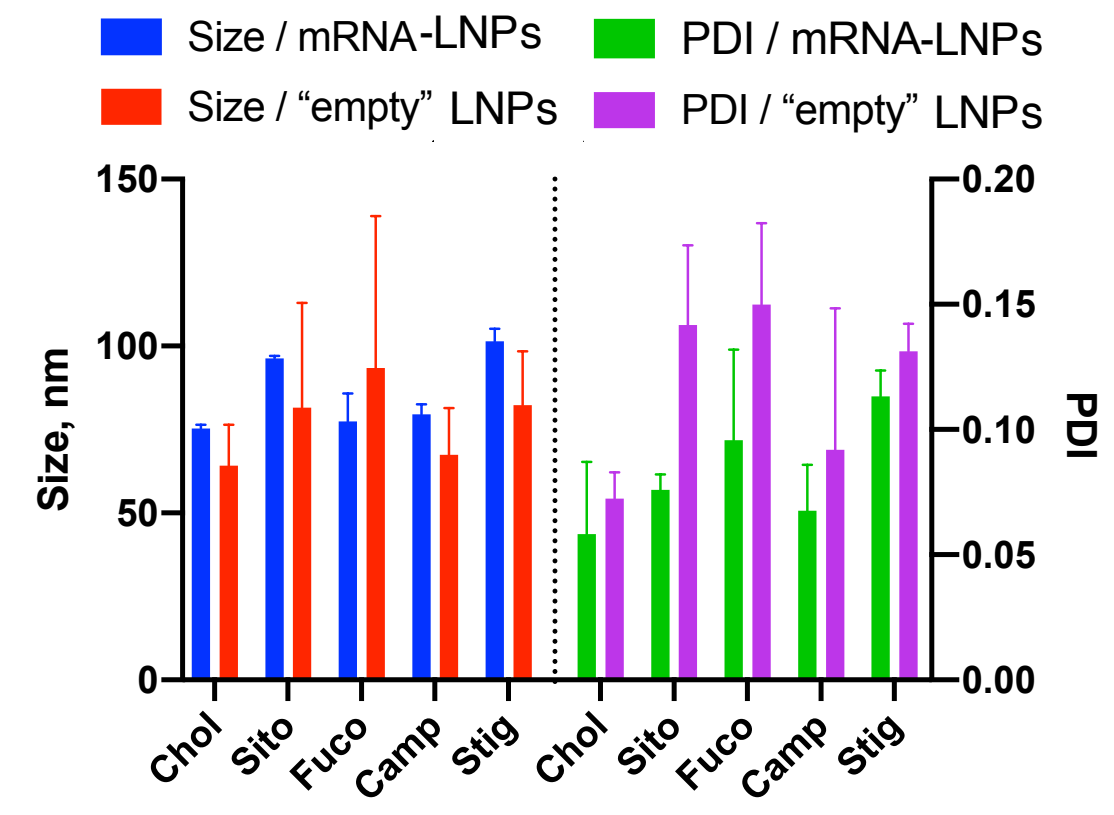

Figure S4: Comparison of DLS data for LNPs with and without mRNA payload ("empty" LNPs were used in DSC experiments $(n=6))$ 


\section{References}

(1) Patel, S.; Ashwanikumar, N.; Robinson, E.; Xia, Y.; Mihai, C.; Griffith, J. P.; Hou, S.; Esposito, A. A.; Ketova, T.; Welsher, K.; Joyal, J. L.; Almarsson, Ö.; Sahay, G. Naturallyoccurring cholesterol analogues in lipid nanoparticles induce polymorphic shape and enhance intracellular delivery of mRNA. Nat. Commun. 2020, 11, 983.

(2) Patel, S.; Ryals, R. C.; Weller, K. K.; Pennesi, M. E.; Sahay, G. Lipid nanoparticles for delivery of messenger RNA to the back of the eye. J. Control. Release 2019, 303, 91-100.

(3) Schindelin, J. et al. Fiji: an open-source platform for biological-image analysis. Nat. Methods 2012, 9, 676-682.

(4) Bernsdorff, C.; Winter, R. Differential Properties of the Sterols Cholesterol, Ergosterol, $\beta$-Sitosterol, trans-7-Dehydrocholesterol, Stigmasterol and Lanosterol on DPPC Bilayer Order. J. Phys. Chem. B 2003, 107, 10658-10664. 